Article

\title{
Overexpression of the Wild Soybean R2R3-MYB Transcription Factor GsMYB15 Enhances Resistance to Salt Stress and Helicoverpa Armigera in Transgenic Arabidopsis
}

\author{
Xin-Jie Shen ${ }^{1, *}+{ }^{\dagger}$, Yan-Yan Wang ${ }^{1,2,+}$, Yong-Xing Zhang ${ }^{1}$, Wei Guo ${ }^{1}$, Yong-Qing Jiao ${ }^{1, *}$ and \\ Xin-An Zhou ${ }^{1, *}$ \\ 1 Key Laboratory of Biology and Genetic Improvement of Oil Crops, Ministry of Agriculture, \\ Oil Crops Research Institute, Chinese Academy of Agricultural Sciences, Wuhan 430062, China; \\ yabofei1212@163.com (Y.-Y.W.); yongxing1008@163.com (Y.-X.Z.); guowei@caas.cn (W.G.) \\ 2 Graduate School of the Chinese Academy of Agricultural Science, Beijing 100081, China \\ * Correspondence: ylssxj@163.com (X.-J.S.); yqjiao@126.com (Y.-Q.J.); zhouocri@sina.com (X.-A.Z.); \\ Tel.: +86-27-8671-2256 (X.-J.S.) \\ + These authors contributed equally to this work.
}

Received: 14 November 2018; Accepted: 5 December 2018; Published: 9 December 2018

\begin{abstract}
Plant R2R3-MYB transcription factors (TFs) have been suggested to play crucial roles in the response to diverse abiotic and biotic stress factors but there is little molecular evidence of this role in soybean plants. In this work, we identified and functionally characterized an R2R3-MYB TF, namely, GsMYB15, from the wild soybean ED059. Protein and promoter sequence analysis indicated that GsMYB15 is a typical R2R3-MYB TF and contains multiple stress-related cis-elements in the promoter region. GsMYB15 is located in the nucleus and exhibits transcriptional activation activity. QPCR assays suggested that the expression of GsMYB15 could be induced by $\mathrm{NaCl}$, insect attacks and defense-related hormones (MeJA and SA). Furthermore, GsMYB15 exhibited highest expression in pods compared to other tissues. Functional analysis of GsMYB15 demonstrated that overexpression of GsMYB15 could increase salt tolerance and enhance the resistance to H. armigera larvae in transgenic Arabidopsis plants. Moreover, overexpression of GsMYB15 also affected the expression levels of salt stress- and defense-related genes in the transgenic plants. Feeding with transgenic Arabidopsis plant leaves could significantly suppress the expression levels of immunity-related genes in $H$. armigera larvae. Overexpression of GsMYB15 also increased mesophyll cell levels in transgenic plants. Taken together, these results provide evidence that GsMYB15 is a positive regulator of salt stress tolerance and insect resistance in transformed Arabidopsis plants.
\end{abstract}

Keywords: R2R3-MYB transcription factor; wild soybean; Helicoverpa armigera; salt stress; Arabidopsis thaliana

\section{Introduction}

As sessile organisms that, unlike animals, cannot escape, plants have evolved sophisticated regulatory mechanisms for the maintenance of growth and development under diverse environmental stress conditions [1-3]. An increasing number of studies have suggested that plants can survive under different stress conditions, most likely by controlling complex plant hormone response networks and molecular signal transduction pathways [4-6]. When they encounter stressful conditions, plants first perceive stress signals via receptors and then exhibit specific changes in gene expression, protein synthesis, metabolic secretion and even physiological structure, leading to stress tolerance [7-9]. 
Among these molecular and physiological factors, the regulatory genes in the nucleus and the encoded proteins are critical for stress response and the functions of these biomolecules can be amplified via signal transduction cascades $[5,10,11]$.

Plant growth and development are mainly regulated by six major hormones or groups of hormones, namely, abscisic acid (ABA), auxin (IAA), gibberellins (GAs), cytokinins (CTKs), ethylene (ET) and brassinolides (BRs). Although their levels are very low in plants, plant hormones play essential roles throughout the plant growth period. In addition to functioning as growth regulators, plant hormones also play important roles in the response to multiple environmental stress factors by acting as stress signaling molecules to transmit stress signals to downstream response genes $[4,6,10]$. The ABA signaling pathway is central to drought and salt stress responses in plants [6]. When plants encounter stressful conditions, the ABA receptors PYLs can sense signals and form the PYL-ABA-PP2C complex and the PP2C-SnRK complex dissociates, leading to the activation of downstream stress-related gene expression by SnRKs [12]. Recent research has also demonstrated that ABA can enhance plant pathogen immunity by regulating stomatal closure via the AtPeps-PEPR signaling pathway [13], indicating that ABA probably has multiple functions and can participate in crosstalk with other plant hormone pathways. Jasmonic acid (JA) and salicylic acid (SA) are the major defense hormones involved in the activation of defensive responses against herbivorous insects and pathogens, respectively [14-16]. When plants are injured by insect attacks, the wound signal stimulates plant JA synthesis and the JA signal then triggers the COI1-JAZ interaction, which leads to the degradation of JAZs by the $26 \mathrm{~S}$ proteasome and to activation of downstream defense-related gene expression, such as the expression of MYC transcription factors (TFs) [17]. A recent study demonstrated that the ET-signaling pathway plays a central role in regulating the resistance to corn leaf aphids (CLAs) in Zea mays leaves but this mechanism is independent of JA [18]. BRs have also been reported to play essential roles in plant-herbivorous insect interactions due to the diverse secondary metabolites of BRs, such as cholesterol; insects must obtain sterol precursors from plants for de novo synthesis and these compounds play a crucial role in insect growth [19]. GAs and CTKs often act as nodes of crosstalk among different plant hormones in the stress response network [20]. For instance, the DELLA proteins can interact with JAZ1 to downregulate downstream defense-related gene expression of the JA signaling pathway [21]. The CTK-activated factor ARR2 can interact with TGA3 to promote gene expression of the SA signaling pathway gene expression, leading to activation of an NPR1-mediated defense mechanism [22].

A growing number of studies have shown that TFs play central roles in regulating gene expression in response to abiotic and biotic stress factors, such as low temperatures, salt, drought and pathogen and insect attacks [3,23-27]. The plant MYB TFs have been demonstrated to be involved in the response to various environmental stress factors and increase plant stress tolerance by activating downstream stress-related signal transduction pathways $[3,28,29]$. There are four types of MYB TFs in plants, namely, R1-, R2R3-, R1R2R3- and 4R-MYB, based on the number of DNA-binding domains [28]. The plant R2R3-MYB TF family has been widely studied in many plants and has been shown to be involved in stress response. In addition, increasing evidence suggests that R2R3-MYB TFs likely have multiple functions in the regulation of different stress responses. Heterologous expression of PacMYBA in Arabidopsis plants can enhance salt tolerance and pathogen resistance [3]. Overexpression of TaMYB1D in tobacco plants can increase the tolerance to drought and oxidative stress by affecting phenylpropanoid metabolism [30]. AtMYB96 can regulate cuticular wax biosynthesis under drought conditions, leading to increased plant drought tolerance [23,31]. AtMYB96 can also enhance pathogen resistance by promoting SA biosynthesis in Arabidopsis. In addition to improving abiotic stress tolerance and pathogen resistance, plant R2R3-MYB TFs also play important roles in the defensive response to insects. TaMYB19, TaMYB29 and TaMYB44 are co-regulators of phloem-based defense against English grain aphids in wheat [32]. AtMYB75 can modulate flavonoid metabolites, leading to resistance against Pieris brassicae via regulation of kaempferol-3,7-dirhamnoside biosynthesis [33]. 
Soybean is one of the most important oil crops in the world. Soybean not only produces cooking oil but also produces high-quality vegetable protein, which are benefic for human health [34]. As global warming increases and indiscriminate use of pesticides, the yield of soybean often face extremely environment conditions and fatal damage by insects [35]. Using molecular biology techniques could create GM soybeans which significantly increase the stress tolerance and yield of soybean [36]. However, the candidate genes using for improving soybeans are still limited. Plant R2R3-MYB TFs have been suggested playing crucial roles in regulating and improving plant in response to multiple abiotic and biotic stresses [2,3,37]. Identify and function analysis more R2R3-MYB TFs might benefit for soybean molecular breeding.

Although the functions of plant R2R3-MYB TFs in stress response have been widely studied in many plants, the role of R2R3-MYB TFs in salt tolerance and insect resistance in soybean plants remains unclear. In this work, we report a wild soybean R2R3-MYB TF, named GsMYB15 and demonstrate that GsMYB15 is a nuclear protein with transcriptional activation activity. Moreover, we showed that GsMYB15 plays a positive role in the response to salt stress and insect resistance in transgenic Arabidopsis plants.

\section{Results}

\subsection{Clone and Sequence Analysis of GsMYB15}

To clone and characterize the wild soybean MYB TF that plays a crucial role in the response to biotic and abiotic stress, we analyzed the transcriptomic data using ED059 to study cotton bollworm resistance. After analyzing the transcriptomic data, we found that the MYB-like gene was significantly upregulated after cotton bollworm feeding in the resistant soybean cultivar ED059 compared to the control susceptible soybean cultivar. Therefore, we designed a pair of specific primers based on the genomic DNA sequence of ED059 to amplify the MYB gene using PCR. We obtained a single putative R2R3-MYB gene product and the product, which we named GsMYB15, shared high homology with the gene AtMYB15. The 939-bp amplification product was the full-length cDNA of GsMYB15 and was located on chromosome 12 of ED059. The predicted protein GsMYB15 contained 313 amino acids, with an isoelectric point of 5.62, an aliphatic index of 72.27, an instability index of 41.95 and a calculated molecular mass of $35 \mathrm{kDa}$ (Supplementary Table S2). After alignment of the amino acid sequence of GsMYB15 with those of other stress-related MYB TFs, GsMYB15 was shown to contain a typical R2R3-MYB functional domain (Figure 1a) and shared the highest similarity with glycine max GmMYB15 (100\% amino acid identity in the R2R3 domain and 99\% identity over the whole coding sequence). Furthermore, a functional motif that is required for the interaction between MYB and bHLH proteins was also identified in the R3 domain of GsMYB15 (Figure 1a). The phylogenetic tree also demonstrated that GsMYB15 was closely related to GmMYB15 (Figure 1b). These results suggested that GsMYB15 might also have similar functions as R2R3-MYBs, which are known to be related to biotic or abiotic stress.

The GsMYB15 gene was then amplified from the genomic DNA of ED059 by PCR using specific primers. The gene structure model in Figure 1c revealed that the GsMYB15 clone, with the approximately $3.7 \mathrm{~kb}$ full-length gene sequence, included three exons and two introns. The R2 domain was present in both the first and second exons, while the R3 domain was present in only the second exon (Figure 1c).

Motif analysis of the 1.8-kb region of the promoter sequence of GsMYB15 revealed seven types of stress-related elements (Table 1). Interestingly, the presence of two MeJA-responsive elements, with the core sequences CGTCA and TGACG, suggested that GsMYB15 might be involved in the development of insect-plant interactions. Moreover, ARE and O2-site motifs, which are involved in anaerobic induction and the regulation of zein metabolism, respectively, were also identified in the promoter region of GsMYB15 (Table 1). 
(a)

(b)
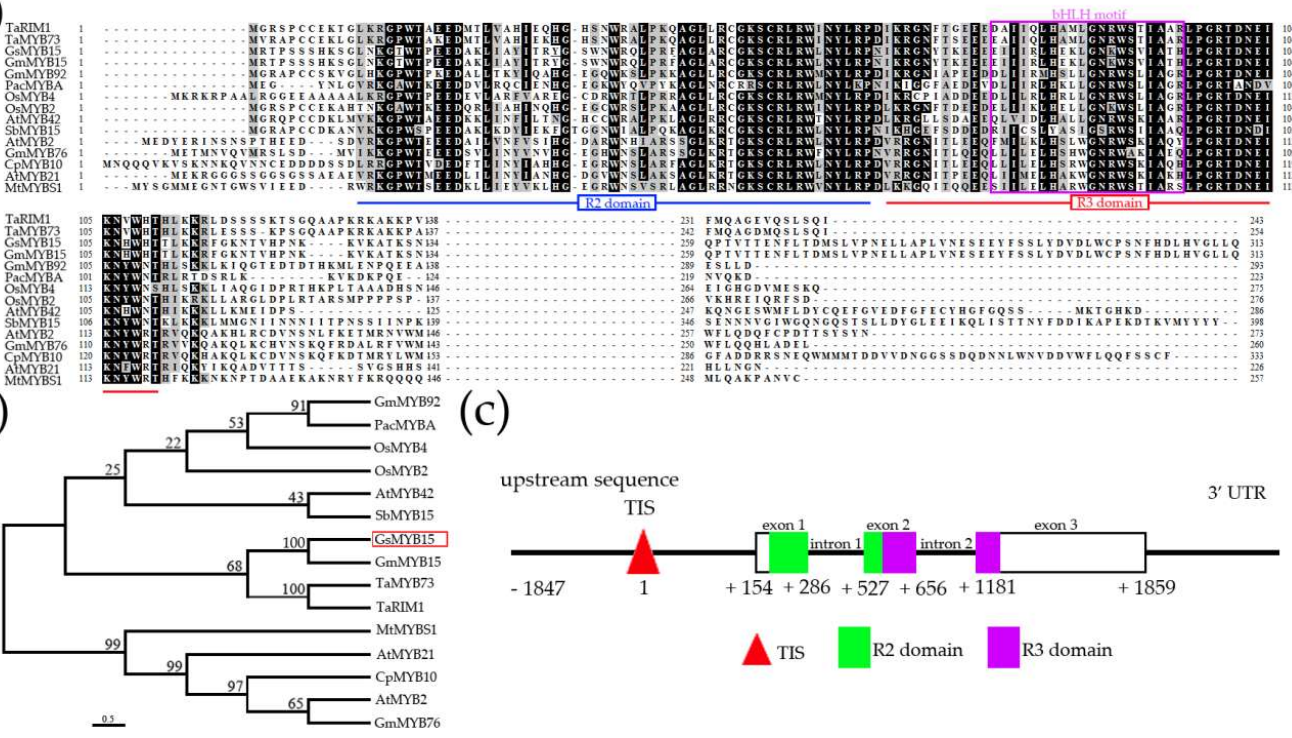

(c)

Figure 1. Phylogenetic relationship between the protein sequences of GsMYB15 and stress-related R2R3 MYBs from other species and genomic sequence analysis of GsMYB15. (a) Protein sequence alignment of GsMYB15 with known stress-related R2R3 MYBs from other species. The R2R3-binding domain is underlined. Green boxes indicate specific residues from the motif implicated in the bHLH cofactor interaction in Arabidopsis [38]. The accession numbers of these proteins in the GenBank database are as follows: PacMYBA (KF974774), AtMYB42 (AEE83118), AtMYB2 (BAA03534), OsMYB4 (BAA23340), OsMYB2 (BAA23338), CpMYB10 (AAM43912), MtMYBS1 (AES60982), SbMYB15 (AKP07635), TaMYB73 (AEW23186), GmMYB15 (Glyma.12g199200), TaRIM1 (AMP18876), AtMYB21 (EFH51661), GmMYB76 (Glyma.02g009800), GmMYB92 (Glyma.16g023000) and GsMYB15 (MH796674). (b) Phylogenetic tree of GsMYB15 and other stress-related R2R3 MYB proteins from diverse species. The scale bar indicates 0.5 substitutions per site. (c) Structure of the GsMYB15 gene sequence. The promoter, introns and 3'UTR are indicated by lines. The transcription initiation site (TIS) and the R2R3 MYB domains are shown as triangles and solid boxes, respectively. Numbers refer to the position relative to the TIS of GSMYB15.

Table 1. Cis-acting elements potentially associated with the stress response of GsMYB15.

\begin{tabular}{ccccc}
\hline Motif & Stand & $\begin{array}{c}\text { Distance } \\
\text { from ATG }\end{array}$ & Sequence & Function \\
\hline \multirow{2}{*}{ ARE } & - & 377 & TGGTTT & Cis-acting regulatory element essential for \\
the anaerobic induction
\end{tabular}




\subsection{Sub-Cellular Localization, Transcriptional Activation Activity and Tissue-Specific Expression of GsMYB15}

To assess the sub-cellular localization of GsMYB15, we generated a recombinant plasmid containing a fusion of GsMYB15 with a reporter gene (GsMYB15-GFP). This plasmid was introduced into tobacco cells and the fluorescence of the fusion protein, which was visualized by a confocal microscope, was observed to be localized to the nucleus (Figure 2a). This result indicated that GsMYB15 may function as a TF.

In this study, we used the Y2H Gold yeast system, which harbored four reporter genes (HIS3, ADE2, AUR1-C and MEL1), to detect the transcriptional activation activity of GsMYB15. All transformants, including the negative control (pGBKT7), positive control (pGAL4) and experimental group (pGBKT7GsMYB15), grew well on SD/Trp medium (Figure 2B). However, only the positive control and experimental group survived on $\mathrm{SD} /-\operatorname{Trp} / \mathrm{His} / \mathrm{X}-\alpha$-Gal medium and the yeast cells turned blue (Figure 2b). In contrast, the negative control did not grow on SD/-Trp/-His/X- $\alpha$-Gal medium (Figure 2b). Therefore, our results indicated that GsMYB15 might have transcriptional activation activity.

To study the tissue localization of GsMYB15, GsMYB15Pro2000-GUS-transformed Arabidopsis plants were grown under normal conditions and used for GUS staining. As shown in Figure 2c, GsMYB15 Pro2000-GUS expression occurred in the roots, leaves and stems of transgenic Arabidopsis plants and was significantly strong in the pods and flowers. No expression was detected in the seeds.

Expression patterns of GsMYB15 in different tissues and organs of wild soybean ED059 were detected under normal conditions (Figure 2d). The results showed that GsMYB15 was expressed in all the organs and tissues examined, including roots, stems, leaves, flowers and pods. GsMYB15 expression was highest in the pods, followed by flowers, roots, stems and leaves, indicating that GsMYB15 is expressed at higher levels in reproductive tissues than in nutritive organs and may function as an inducible transcription factor (Figure 2d).

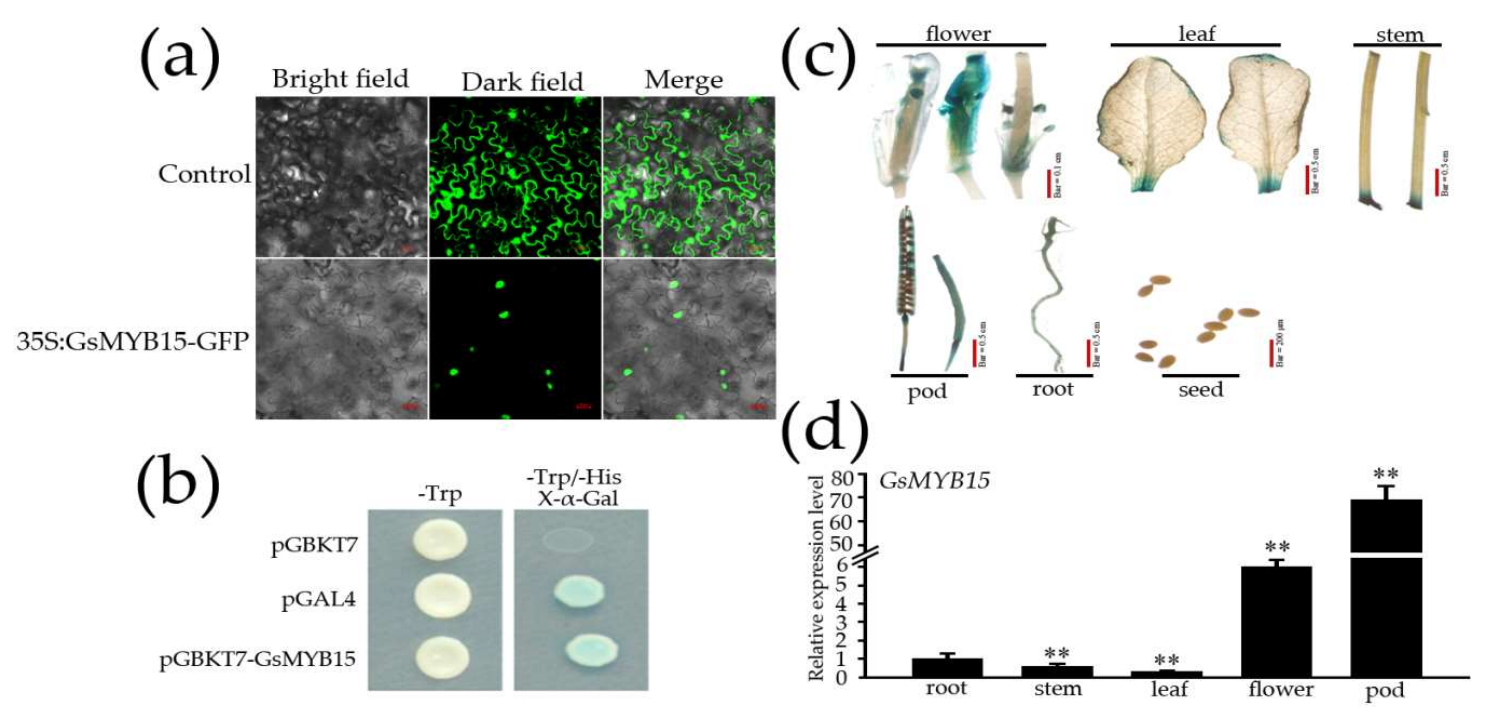

Figure 2. Sub-cellular localization, transcriptional activation, GUS staining and tissue-specific expression of GsMYB15. (a) Control, transient expression of the PJL12m-GFP vector in tobacco leaves; 35:GsMYB15-GFP, transient expression of the PJL12m-GsMYB15-GFP vector only in tobacco leaves. (b) pGBKT7, negative control; pGBKT7-GSMYB15, self-activation of transcription; pGAL4, positive control. (c) Histochemical staining of GUS in Arabidopsis plants transformed with GsMYB15pro::GUS. (d) RT-PCR analysis of GsMYB15 expression in the roots, stems, leaves, flowers and pods. SKIP16 was used as the internal reference control. Each point represents the mean value of three independent experiments performed in triplicate \pm SE. Statistically significant differences were assessed using Student's $t$-test $(* * p<0.01)$. 


\subsection{Expression of GsMYB15 in Soybean Leaves in Response to Salt, MeJA and SA Treatments and Insect Attacks}

To investigate whether GsMYB15 is involved in salt stress and insect-plant interactions, we examined the expression patterns of GsMYB15 in the wild soybean ED059 seedlings after exposure to salt stress and to the biotic stress-related plant hormones SA and MeJA. Moreover, we also analyzed the expression patterns of GsMYB15 in the resistant wild soybean ED059 and susceptible soybean Tianlong 2. The results revealed that salt stress significantly induced GsMYB15 expression in soybean leaves after $1 \mathrm{~h}$ of salt treatment and gradually upregulated expression for up to $6 \mathrm{~h}$ of salt treatment, attaining levels that were 4 -fold greater than those observed before treatment; the expression levels decreased after $12 \mathrm{~h}$ of salt treatment (Figure 3a). QPCR results also showed that GsMYB15 expression could be induced gradually by both MeJA and SA (Figure 3b). The expression level of GsMYB15 was also significantly upregulated after $H$. armigera larval attack in the resistant wild soybean ED059 compared with the susceptible soybean Tianlong 2 (Figure 3c). Moreover, the pathogen-related phytohormone SA could induce the expression of GsMYB15, similar to MeJA (Figure 3d). These results indicated that GsMYB15 could respond to salt, MeJA and SA treatments and insect attacks.

(a)

(c)
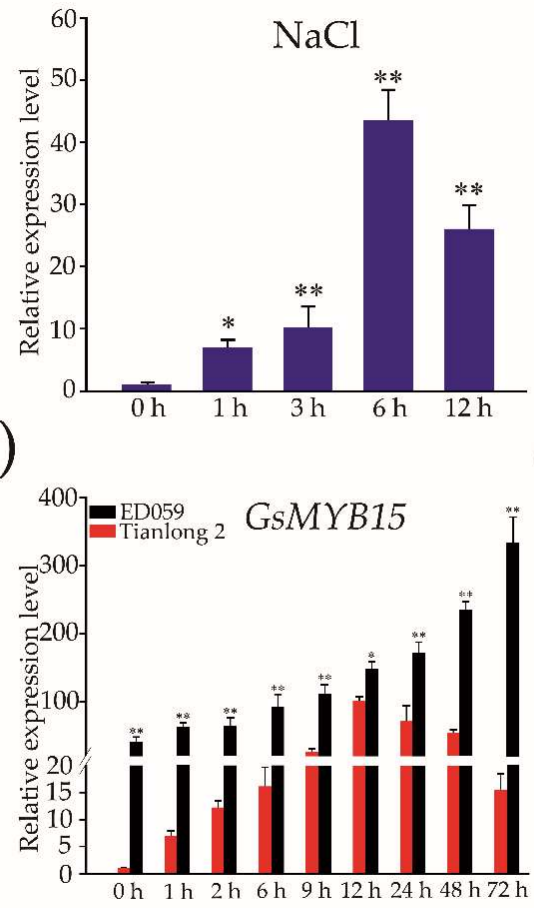

(b)

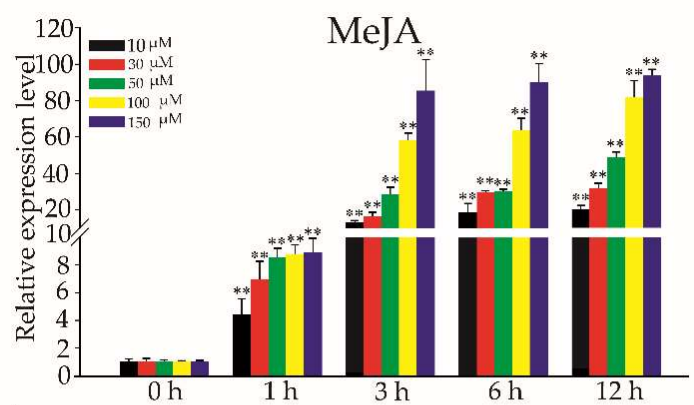

(d)

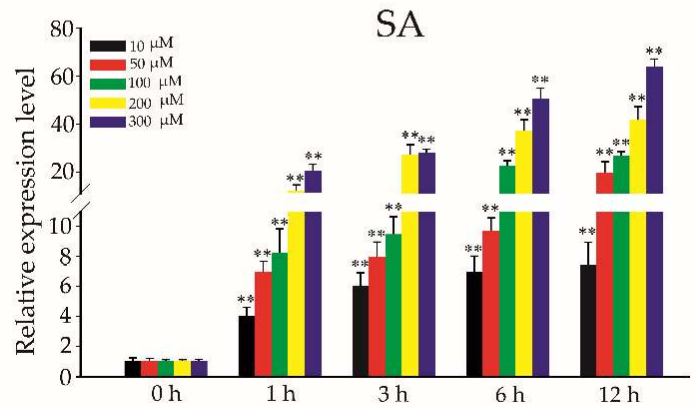

Figure 3. Expression patterns of GsMYB15 in soybean in response to $\mathrm{NaCl}(\mathbf{a}), \mathrm{MeJA}(\mathbf{b}), \mathrm{SA}$ treatments (c) and H. armigera larvae (d). Plants were collected at the indicated time points. GsSKIP16 was used as the internal reference control to normalize the templates. The relative mRNA levels are represented as the mean $\pm \mathrm{SD}(n=3)$. Statistically significant differences were assessed using Student's $t$-test $\left.{ }^{*} p<0.05,{ }^{* *} p<0.01\right)$.

\subsection{Physiological Changes in Arabidopsis Lines Overexpressing GsMYB15}

To prove the generation of GsMYB15 transgenic Arabidopsis plants, we use PCR method to amplification the coding sequence of GsMYB15 in both transgenic lines and wild type Arabidopsis. As results shown in Figure 4a, bands only exist in agars which using the transgenic generations DNA as amplification templates and no band exist in wild type Arabidopsis (Figure 4a). Moreover, the results of immunostrip, which is specifically for detections of the protein of glufosinate-ammonium also demonstrating that only transgenic Arabidopsis generations could show two bands while the wild type 
Arabidopsis only have one band (Figure 4a). In this work, three $\mathrm{T}_{3}$ GsMYB15-overexpressing Arabidopsis lines (L-2, L-3 and L-4) with relatively high expression levels were used to investigate the function of GsMYB15 in plant biotic and abiotic stress responses (Figure 4b). Under normal conditions, no obvious phenotypic differences were observed between the control and transgenic plants. However, the results of paraffin sectioning showed that the transgenic plants (L-4) had more mesophyll cells in the leaf tissues than the control plants (Figure 4c). The results also showed that the transgenic plants had more xylem cell in both the main stem and side stem tissues than the control plants (Figure $4 \mathrm{~b}$ ). The other two transgenic plants (L-2 and L-3) also have the similar phenotypes as L-4 in leaf tissues, main stem and side tissues (date not shown).

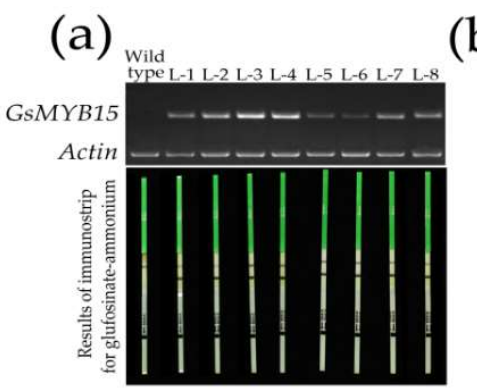

(b)

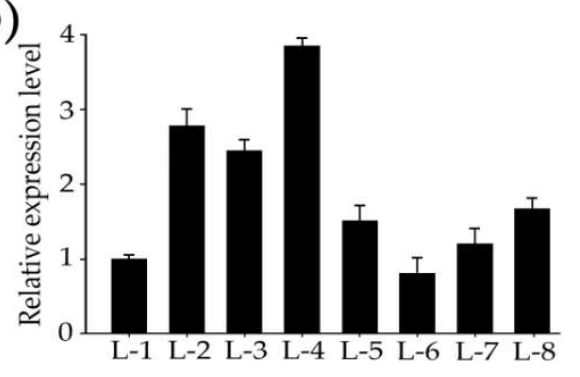

(c)

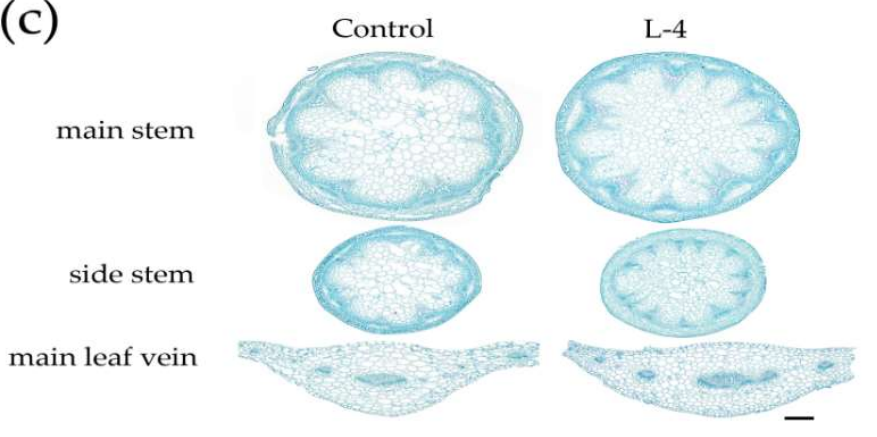

Figure 4. Analyses of GsMYB15 transgenic plants grown in soil. (a) Prove GsMYB15 transgenic Arabidopsis generations using semi-quantitative RT-PCR and immunostrip methods. (b) Expression levels of GsMYB15 in different transgenic Arabidopsis lines. GsSKIP16 was used as the internal reference control. Each point represents the mean value of three independent experiments performed in triplicate \pm SE. (c) Four-week-old rosette leaves, main stems and side stems from the top were chosen for transverse section analysis. Bar $=200 \mu \mathrm{m}$.

\subsection{Overexpression of GsMYB15 Increased Salt Stress Tolerance in the Transgenic Arabidopsis Lines}

To assess the effect of GsMYB15 overexpression on salt tolerance in Arabidopsis plants during the seed germination stage, seeds of control and transgenic plants were sown and germinated on MS medium with or without high-salt treatment. As shown in Figure 5a, there was no phenotypic difference between the control and transgenic seeds during seed germination and seedling development under normal MS medium conditions. After 3 days of treatment in high-salt MS medium (containing $100 \mathrm{mM} \mathrm{NaCl}$ ), the germination of the control seeds was markedly suppressed (Figure $5 \mathrm{a}$ ). However, compared to the control seeds, the seeds from the transgenic plants that heterologously expressed GsMYB15 exhibited markedly increased germination percentages (Figure 5a). Moreover, after 7 days of growth on high-salt MS medium, most of the transgenic plants continued to develop and the leaves remained green after seed germination (Figure 5a). The germination rates of the three transgenic lines treated with $100 \mathrm{mM} \mathrm{NaCl}$ ranged from 64 to $71 \%$ (Figure $5 \mathrm{~b}$ ).

We next transferred 12-day-old seedlings of the control and transgenic plants from MS medium to soil conditions and grew the seedlings for four weeks to further investigate the salinity tolerance of the transgenic plants during seedling development. Under normal conditions, there were no 
obvious phenotypic differences between the control and transgenic plants (Figure 5c). After 7 days of treatment with $150 \mathrm{mM} \mathrm{NaCl}$, the control plants exhibited chlorosis in the leaves and growth retardation compared to the transgenic plants (Figure 5c). However, the transgenic plants remained green and grew well after 7 days of treatment (Figure 5c). When treated with $250 \mathrm{mM} \mathrm{NaCl}$, the control plants completely stopped growing and the leaves also turned white and wilted (Figure $5 \mathrm{c}$ ). In contrast, although the transgenic plants became weak and exhibited leaves with slight chlorosis, most remained alive and continued to grow (Figure 5c). The survival rates of the three transgenic lines treated with $250 \mathrm{mM} \mathrm{NaCl}$ ranged from 42 to $53 \%$ (Figure $5 \mathrm{~d}$ ). These results indicated that GsMYB15-overexpressing Arabidopsis lines exhibited enhanced tolerance to salt stress.

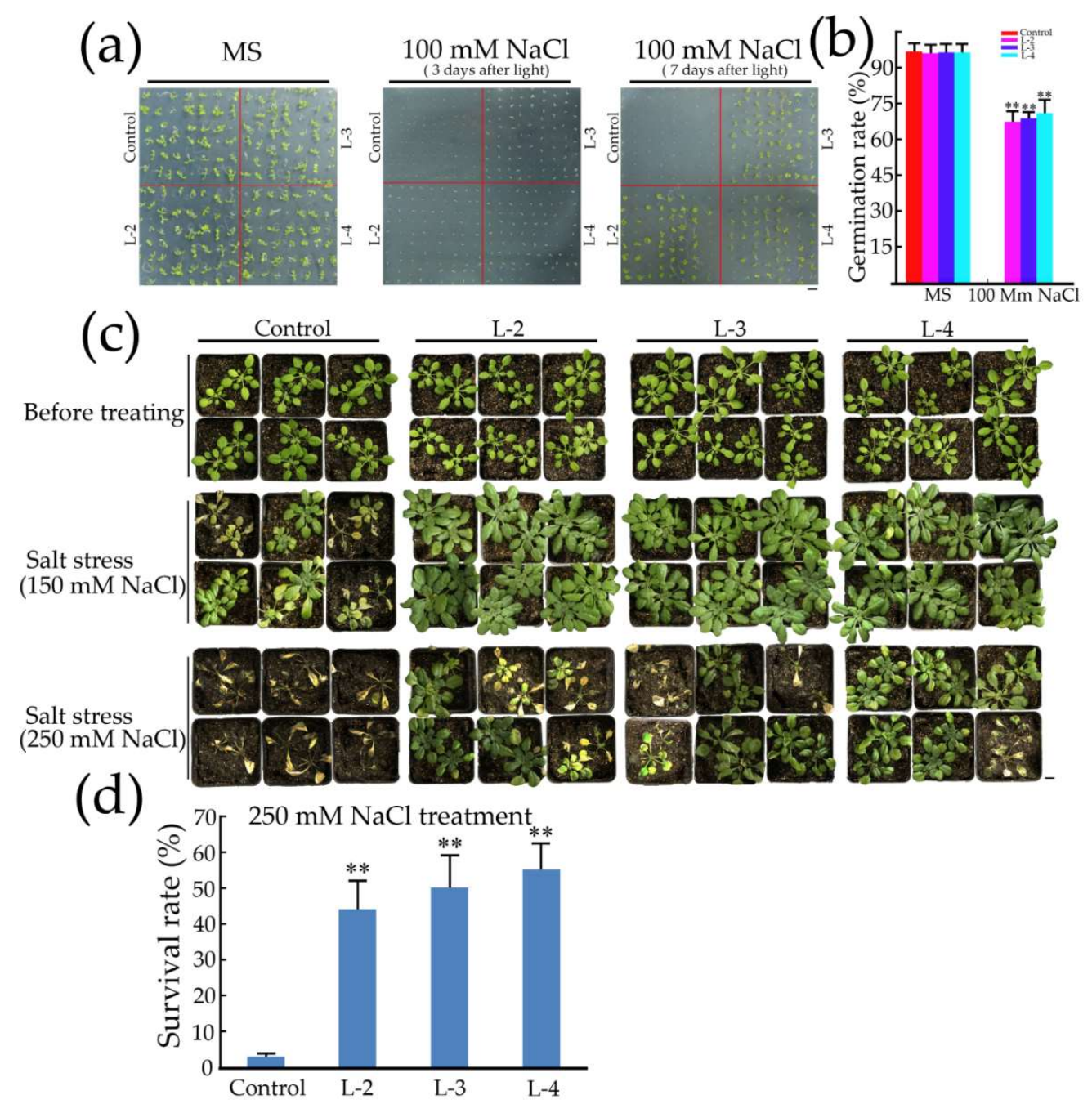

Figure 5. Comparative analysis of seed germination and seedling survival rate under salt stress among control (Col-0) and transgenic Arabidopsis plants. (a) Germination status of control, L-2, L-3 and L-4 seeds in MS plates, with or without supplementation with $100 \mathrm{mM} \mathrm{NaCl}, 3$ or 7 days after exposure to light. Bar $=1 \mathrm{~cm}$. (b) Germination rate of Col-0, L-2, L-3 and L-4 seeds in MS plates, with or without supplementation with $100 \mathrm{mM} \mathrm{NaCl}, 3$ days after exposure to light. (c) The phenotypes of the control and transgenic Arabidopsis plants under normal and different concentrations (150 mM and $250 \mathrm{mM}$ ) of $\mathrm{NaCl}$. Four-week-old seedlings grown in soil were assessed. Bar $=1 \mathrm{~cm}$. (d) Survival rates of transgenic Arabidopsis plants treated with $250 \mathrm{mM} \mathrm{NaCl}$. Error bars indicate SDs. Statistically significant differences were assessed using Student's $t$-test $\left({ }^{* *} p<0.01\right)$. 


\subsection{Expression Pattern Analysis of Salt-Related Genes in Transgenic Arabidopsis Plants}

To study the molecular regulatory mechanism of GsMYB15 in the enhancement of salt tolerance in transgenic Arabidopsis plants, we selected several salt-stress-related genes, such as ABA pathway markers (AtABI1, AtABI2 and AtSnRK2.4), WRKY TFs (AtWRKY25, AtWRKY33 and AtWRKY46) and other salt stress response genes (AtRD20, AtRD26, AtRD29B, AtDREB2A, AtMYB2, AtGSTU17, AtANACO19 and AtHAL3) $[1,3,39,40]$ and detected the expression levels of these genes by real-time PCR (QPCR) and compared the control and transgenic Arabidopsis line 4 (L-4). As shown in Figure 6, the expression levels of AtSnRK2.4, AtWRKY25, AtWRKY33, AtWRKY46, AtRD20, AtRD26, AtRD29B, AtDREB2A, AtMYB2, AtANACO19 and AtHAL3 were significantly higher in L-4 than in the control plants. The expression levels of AtABI1 and AtABI2 were lower in the transgenic plants than in the control plant. Moreover, the expression of AtGSTU17 was also lower in L-4 than in the control plants, which indicated that overexpression of GsMYB15 might weaken glutathione (GSH) biosynthesis and affect several stress-related hormone pathways in transgenic plants. These results indicated that overexpression of GsMYB15 increased the salt tolerance of transgenic plants by affecting several stress-related hormone signal transduction pathways and the expression of some key stress-related TFs.

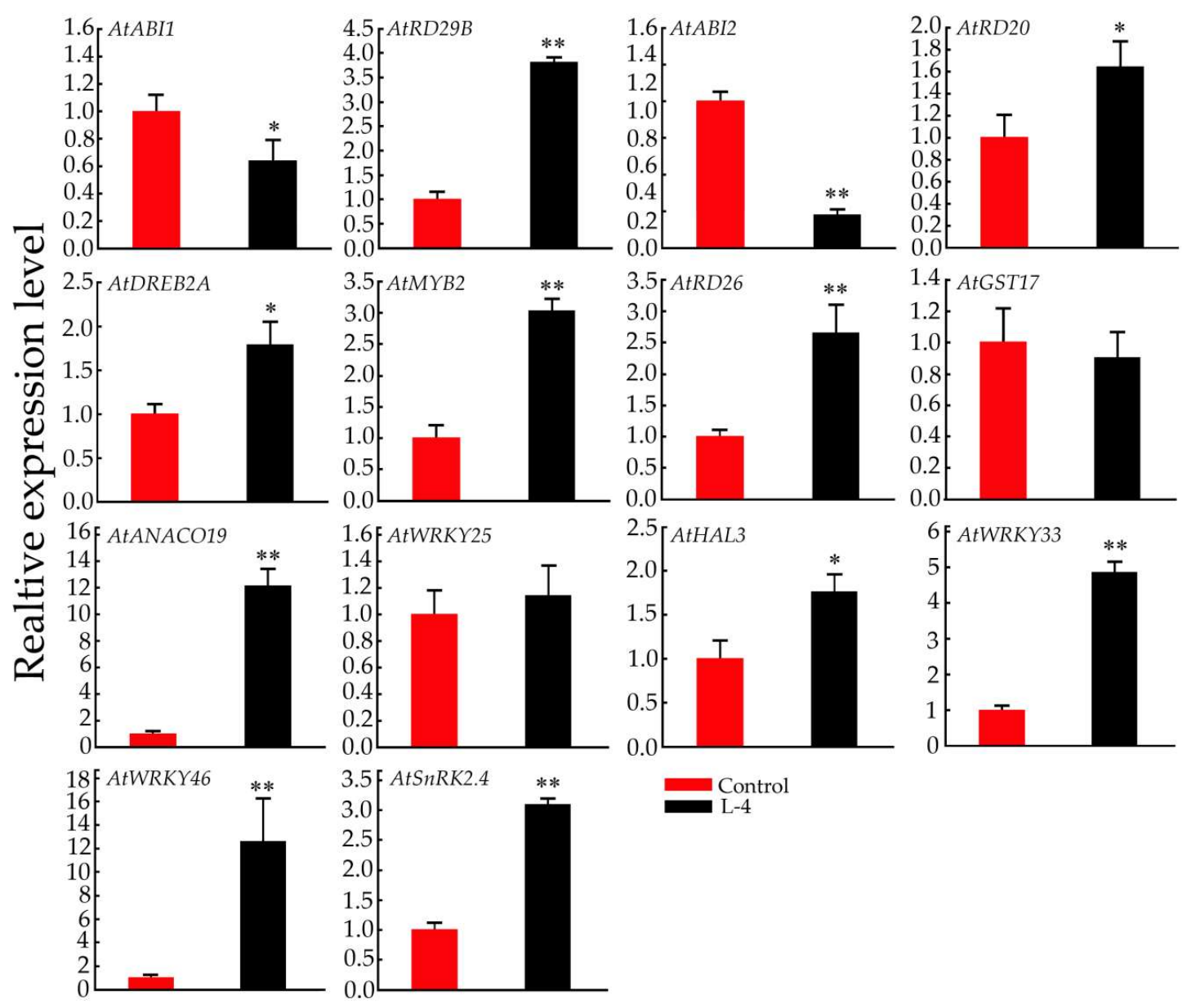

Figure 6. QPCR analysis of the expression levels of salt stress-related genes in the control (Col-0) and transgenic (L-4) Arabidopsis plants. Arabidopsis actin 1 (AtActin1) was used as an internal standard to normalize the templates. The relative mRNA levels are represented as the mean $\pm \operatorname{SD}(n=3)$. Statistically significant differences were assessed using Student's $t$-test $\left({ }^{*} p<0.05,{ }^{* *} p<0.01\right)$.

\subsection{Overexpression of GsMYB15 Increased Insect Resistance in Transgenic Arabidopsis Plants}

Many studies have shown that JA plays a crucial role in plant resistance to insect invasion $[15,41,42]$. Based on the results of cis-element analysis of the GsMYB15 promoter, we found six 
MeJA response elements (Table 1) in the promoter region, indicating that GsMYB15 may be involved in JA-mediated insect resistance. Moreover, it has been shown (Figure 3b,c) that GsMYB15 could respond to insect attack and MeJA treatment. Therefore, we tested the insect resistance ability of the control and transgenic plants heterologously expressing GsMYB15 via a H. armigera larval feeding assay. As shown in Figure 7a,b, both the whole plants and detached leaves of the control plants were significantly chewed by $H$. armigera. In contrast, although the transgenic plants were also chewed by the $H$. armigera larvae, the leaf loss was significantly less than that observed for the control plants. The insect feeding assays further showed that $H$. armigera larvae fed transgenic plant leaves gained significantly less weight increase than those fed control plants (Figure 7c). These results indicated that overexpression of GsMYB15 increased transgenic Arabidopsis plant resistance to insects.

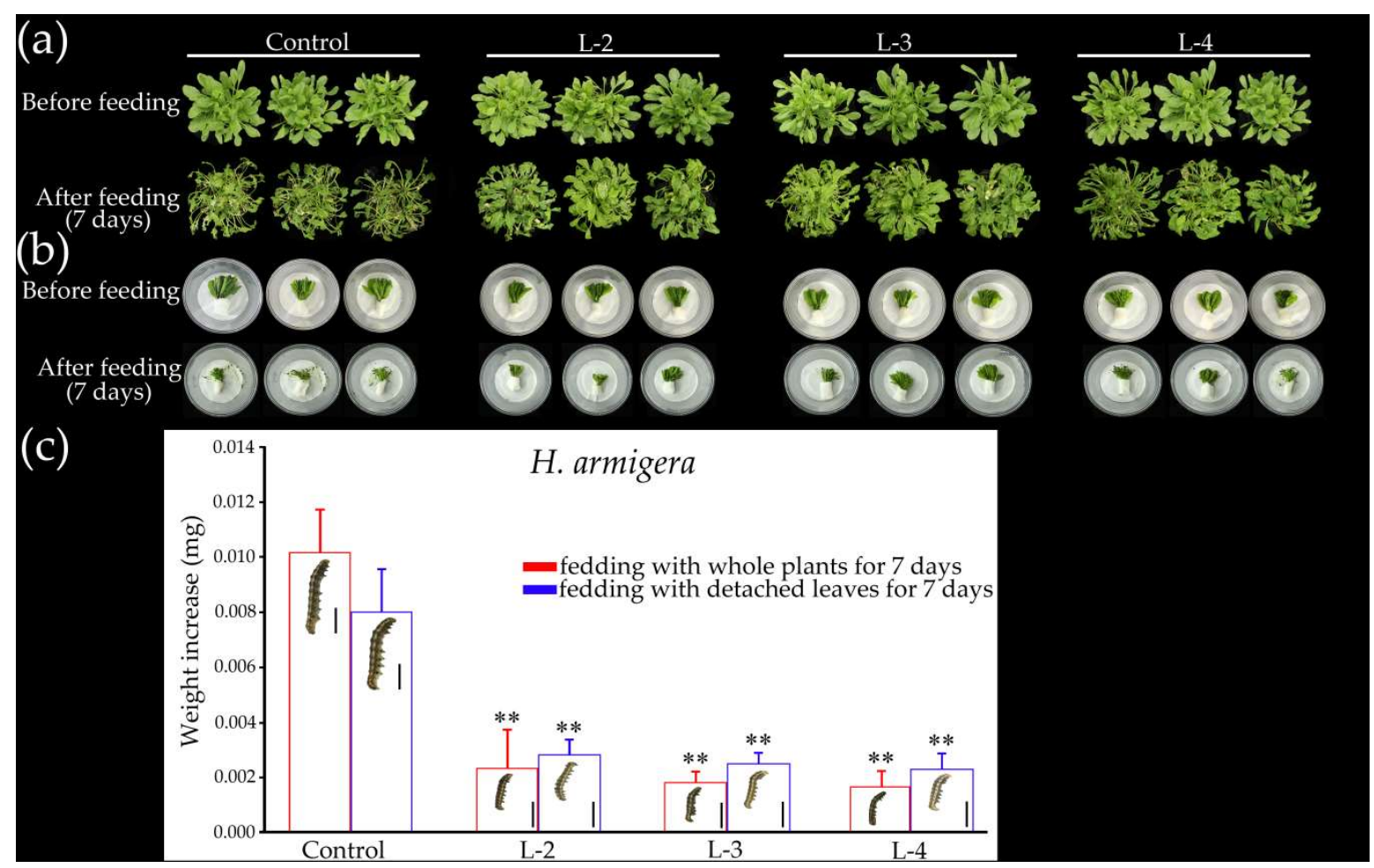

Figure 7. Overexpression of GSMYB15 enhances insect resistance in transgenic Arabidopsis plants. (a) Phenotypes of the control plants (Col-0) and GsMYB15 transgenic plant lines (L-2, L-3 and L-4) before and after $H$. armigera larval attack. (b) Phenotypes of detached leaves from the control plants (Col-0) and GsMYB15 transgenic plant lines before and after feeding with H. armigera larvae. (c) Weight increase of $H$. armigera larvae fed with whole plants or detached leaves from control plants (Col-0) and GsMYB15 transgenic plant lines for 7 days, respectively. Data are the means \pm SDs $(n=15)$. Shown in the column is an image of an H. armigera larva after feeding. Bar $=0.25 \mathrm{~cm}$. Statistically significant differences were assessed using Student's $t$-test $(* * p<0.01)$.

\subsection{Expression Pattern Analysis of Immunity-Related Genes in H. armigera}

The above results suggested that overexpression of GsMYB15 in Arabidopsis plants could increase plant resistance to $H$. armigera larvae (Figure $7 \mathrm{a}, \mathrm{b}$ ). The growth of $H$. armigera larvae fed transgenic plant leaves was significantly weaker than that of larvae fed control plant leaves, indicating that the use of transgenic plant leaves as food might affect the digestive and immune systems and induce toxic side effects in $H$. armigera larvae. Therefore, we used qPCR to examine the expression levels of major immunity-related genes in $H$. armigera larvae fed control or transgenic plant leaves. As shown in Figure 8, the expression levels of all the selected immunity-related genes except HaGali were significantly higher in the larvae fed control plant leaves than in those fed transgenic plant leaves 
(Figure 8). These results suggested that the use of transgenic plant leaves as food may severely damage the immune systems of $H$. armigera larvae and further affect normal growth and development.

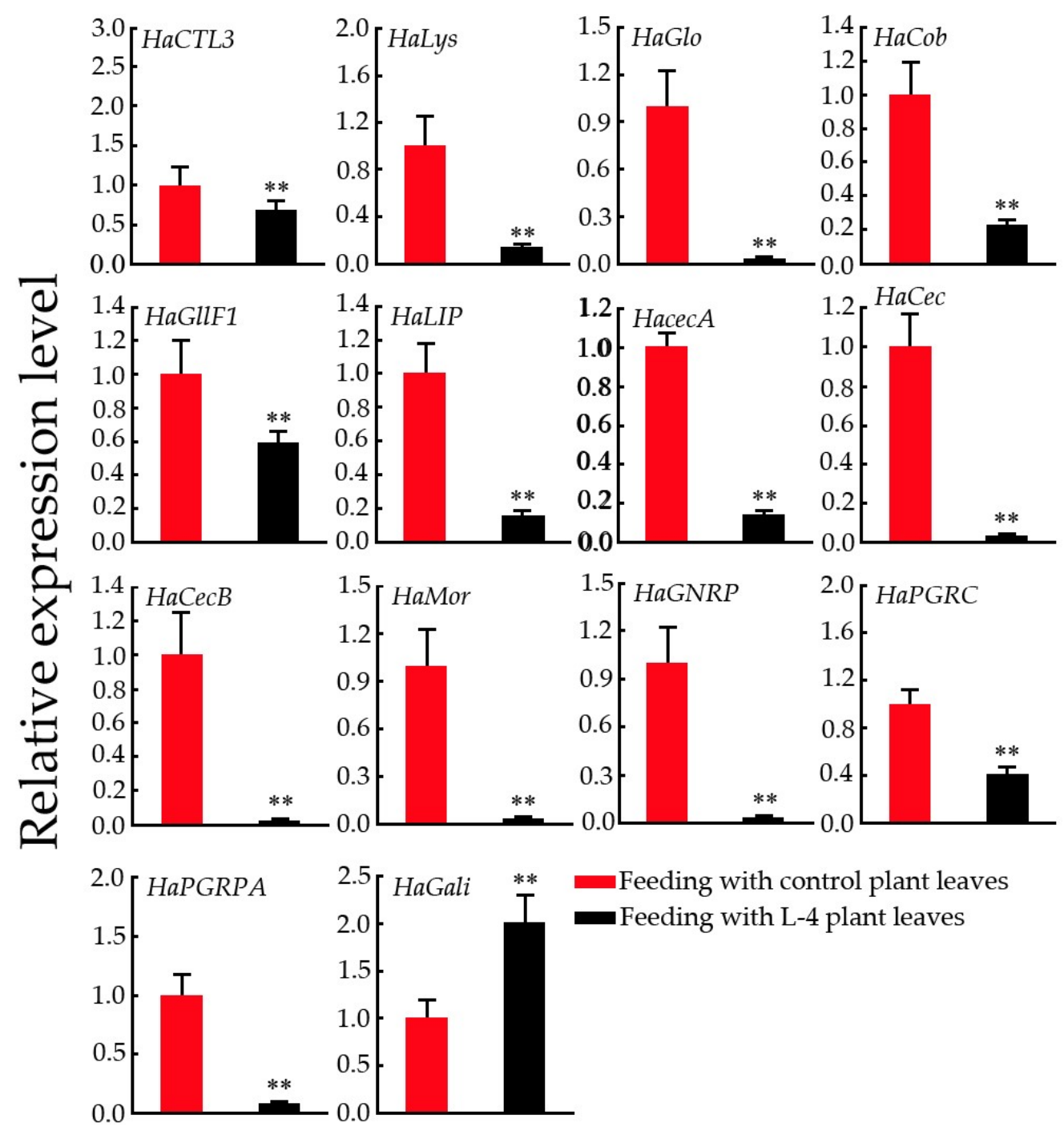

Figure 8. Expression patterns of $H$. armigera immunity-related genes in H. armigera fed wild-type plants leaves (red column) and transgenic Arabidopsis plant leaves heterologously expressing GsMYB15 (black column). H. armigera actin (HaActin) was used as an internal standard to normalize the templates. The relative mRNA levels are represented as the mean $\pm \mathrm{SD}(n=3)$. Statistically significant differences were assessed using Student's $t$-test $(* *<0.01)$.

\subsection{Expression Pattern Analysis of Defense-Related Genes in Transgenic Arabidopsis Plants}

To investigate the enhanced resistance of transgenic plants to insect attacks, we examined the expression patterns of PR genes, JA response genes and other defense-related genes. As shown in Figure 9, the results suggested that overexpression of GsMYB15 could significantly upregulate the expression levels of JA signaling pathway genes, including AtPDF1.2, AtVSP2 and AtLOX2. Other defense-related genes, such as AtEDR1, AtACS6, AtPAD4, AtVSP1, AtAPX1, AtTAT1 and $A t C Y P 79 B 2$, were also upregulated significantly in the transgenic Arabidopsis plants compared with the control plants (Figure 9). In contrast, the expression levels of pathogen-related genes (PR1 and PR5) were downregulated in the transgenic Arabidopsis plants compared to the control plants (Figure 9). Two negative regulators of stress-related genes were significantly suppressed in transgenic plants compared with control plants. 


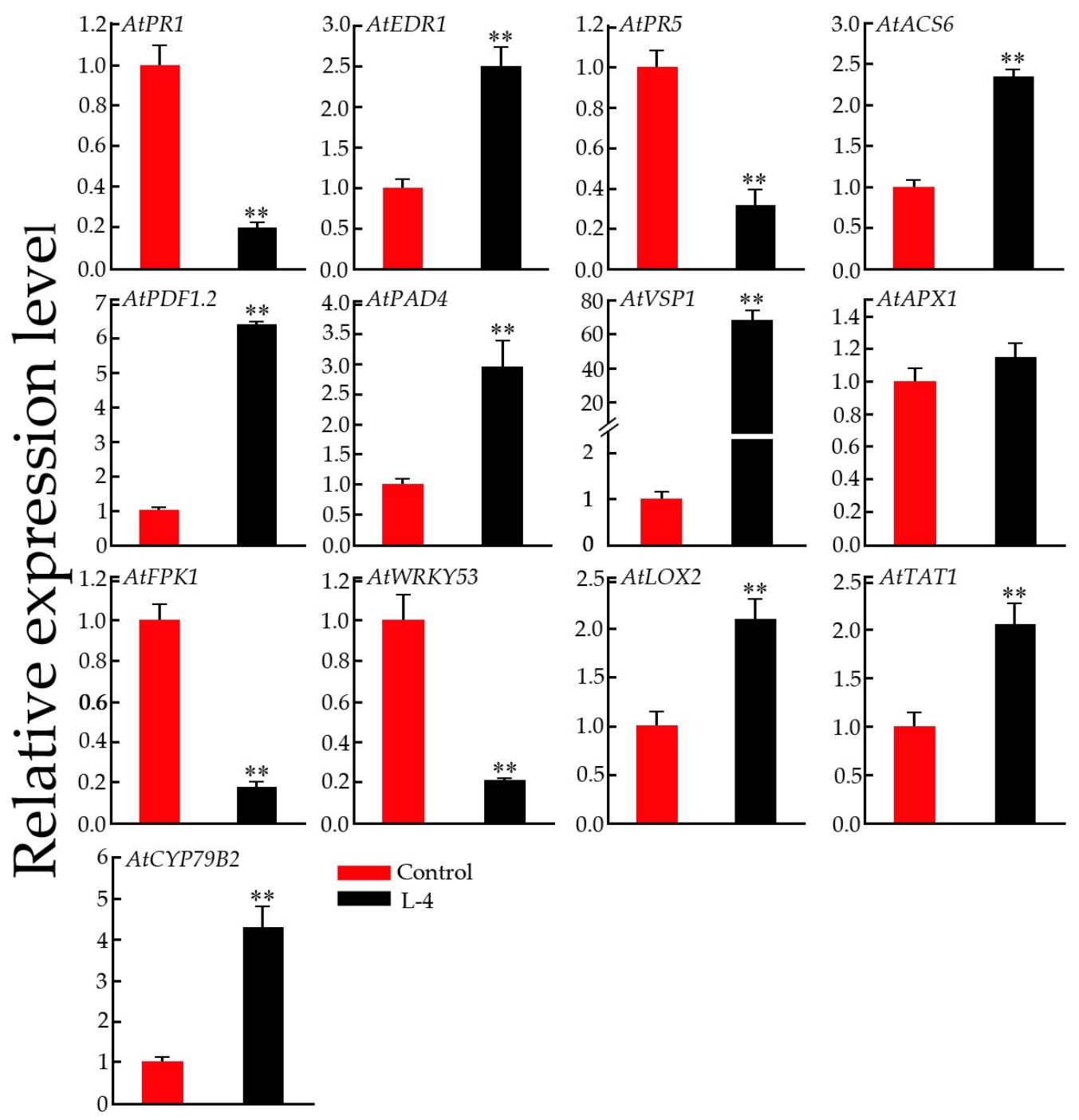

Figure 9. QPCR analysis of the expression levels of defense-related genes in the control (Col-0) and transgenic (L-4) Arabidopsis plants. Arabidopsis actin 1 (AtActin1) was used as an internal standard to normalize the templates. The relative mRNA levels are represented as the mean $\pm \mathrm{SD}(n=3)$. Statistically significant differences were assessed using Student's $t$-test $\left({ }^{* *} p<0.01\right)$.

\section{Discussion}

\subsection{GsMYB15 Can Respond to Salt Stress, MeJA and SA Treatment and Insect Attacks}

Plant MYB-type proteins constitute a large TF family. To date, a number of R2R3-MYB family members have been reported to be involved in the responses of plants to various environmental stress factors in a number of plant species $[1,4,10,30]$. However, to date, very few soybean R2R3-MYB TFs have been reported to be involved in salt stress and the response to herbivorous insect attacks. In the present study, we isolated and functionally characterized an R2R3-type MYB TF, namely, GsMYB15, from the insect-resistant wild soybean ED059. GsMYB15 was significantly upregulated by $\mathrm{NaCl}$, MeJA and SA treatment. However, previous study demonstrated that exogenous application of SA reduces JA biosynthesis and suppresses JA-mediated gene expression reported that a stress-related R2R3-MYB TF PacMYBA also could be induced by both JA and SA treatments at suitable concentrations $[16,43]$. Our results suggested that GsMYB15 might have a similar function to PacMYBA that is involved in stress response. In addition, we identified multiple stress-related cis-elements in the promoter region of GsMYB15, suggesting that GsMYB15 might be regulated by stress signals and other TFs. Furthermore, GsMYB15 was induced by $H$. armigera larval attack and the expression levels of GsMYB15 were 
significantly higher in ED059 than in the susceptible soybean cv. Tianlong 2. These results indicated that GsMYB15 may be involved in both responses to both abiotic stress and plant-insect interactions.

\subsection{GsMYB15 Plays a Positive Regulatory Role in Salt Stress Tolerance in Transgenic Arabidopsis Plants}

The phytohormone ABA and the ABA signal transduction pathways have been demonstrated to play crucial roles in the plant response to salt stress [6,29]. Plants in high-salt conditions generate primary stress signals followed by secondary signals, such as hyperosmotic signals, leading to the accumulation of ABA, which triggers the ABA signaling pathway and activates the expression of downstream stress response genes, finally leading to enhanced plant salt tolerance $[1,12,27]$. In our research, heterologous expression of GsMYB15 in Arabidopsis plants significantly improved plant salt tolerance during high-salt irrigation treatment. This result indicated that GsMYB15 may be associated with the salt stress response. Furthermore, the marker genes (AtABI1 and AtABI2) of negative regulation of the ABA signaling pathway had lower transcript abundance in GsMYB15 transgenic plants than in the control plants, indicating that GsMYB15 may be involved in the ABA-dependent pathway under salt stress conditions. In addition, several stress-related TF family genes were also significantly upregulated in the transgenic plants, such as AtWRKY25, AtWRKY33, AtWRKY46, AtRD20, AtRD26, AtRD29B, AtDREB2A and AtMYB2, all of which have been demonstrated to confer salt stress tolerance in transgenic plants [44-48]. Our results also demonstrated that GsMYB15 had transcriptional activation activity in $\mathrm{Y} 2 \mathrm{H}$ yeast cells, indicating that GsMYB15 may act as a regulator to regulate the expression of other genes or interacting with other TFs as TF complex to affect downstream genes expression. Taken together, these data indicate that GsMYB15 may play a positive role in the plant response to salt stress; however, further investigation is required.

\subsection{GsMYB15 Enhances Herbivorous Insect Resistance in Transgenic Arabidopsis Plants}

In field conditions, plants face diverse types of herbivorous insect attacks during the entire plant growth period. Over a long-term evolutionary period, plants have developed sophisticated and integrated regulatory mechanisms to protect themselves from herbivorous insect attacks [15,49]. When the insects chew or suck the plant tissues with their mouth parts, the injured plant cells at the wound produce initial signals, which are then transmitted within the plant via defense-related signal transduction pathways to trigger the plant immune response, finally leading to the promotion of insect resistance $[2,14,18]$. In these signal transduction networks, TFs play central and essential roles in regulating gene expression by directly or indirectly affecting the promoter regions of target genes $[6,10,28]$. Lots of studies have suggested that plant R2R3-MYB TFs play crucial roles in both abiotic and biotic stresses. Most studies are founding their important functions on environment stresses and plant disease [50-54]. However, very few studies explore their functions in plant-insect interactions, especially in soybean plants. In the present study, a wild soybean R2R3-MYB TF gene, named GsMYB15, we found that the weight increase of H. armigera larvae fed GsMYB15 transgenic plant leaves was less than that of larvae fed control plant leaves, which suggested that heterologous expression of GsMYB15 could enhance the insect resistance of transgenic plants. Many studies have demonstrated that the JA and SA signaling pathways exhibit antagonistic effects [55]. The JA signaling pathway plays a pivotal role in plant defense against insects [2]. The JA signaling pathway can promote the production and accumulation of defense compounds, including glucosinolates, phenolics, proteinase inhibitors and cyanogenic glucosides, in plants $[15,56,57]$. The present work showed that heterologous expression of GSMYB15 could significantly enhance the expression levels of JA response genes such as AtPDF1.2, AtACS6 and AtPAD4 in transgenic plants compared with control plants. Furthermore, our results also suggested that the expression levels of immunity-related genes were significantly decreased in larvae fed GsMYB15 transgenic plant leaves for 7 days, indicating that long-term feeding with GsMYB15 transgenic plant leaves may destroy the immune systems of larvae. Future work should focus on analysis and determination of the primary metabolites that play key roles in insect resistance in GsMYB15 transgenic plants. Interestingly, paraffin sectioning results showed 
that there were more mesophyll cells in GsMYB15 transgenic plant leaves than in the control plants, indicating that these structural changes may have contributed to insect resistance in the GsMYB15 transgenic plants.

\section{Materials and Methods}

\subsection{Soybean Plants and Treatments}

The seeds of the soybean cv. Tianlong 2 and the wild soybean accession ED059 used in this work were obtained from Professor Xinan Zhou (the Institute of Oil Crops Research, Chinese Academy of Agricultural Sciences, Wuhan, China). The seeds were pre-germinated on moistened filter paper in a plant growth chamber at $26{ }^{\circ} \mathrm{C}$ with a $16 \mathrm{~h}$ light $/ 8 \mathrm{~h}$ dark regimen for 5 days. The seedlings were then transferred and grown in Hoagland's nutrient solution until the third compound leaf opened completely. For salt, SA or methyl jasmonate (MeJA) treatment, the seedlings were placed in a $250 \mathrm{mM} \mathrm{NaCl}, 10 / 50 / 100 / 200 / 300 \mu \mathrm{M} \mathrm{SA}$, or 10/30/50/100/150 $\mu \mathrm{M}$ MeJA solution, respectively and collected after $0,1,3,6$, or $12 \mathrm{~h}$. The untreated control contained only Hoagland's nutrient solution. For Helicoverpa armigera larval feeding treatments, the seedlings were collected after 0, 1, 2, 6, 9, 12, 24,48 or $72 \mathrm{~h}$. The seedlings were immediately frozen in liquid nitrogen and stored at $-80^{\circ} \mathrm{C}$ until further analysis. All the treatments were performed in biological triplicates.

\subsection{Sequence Analysis}

The 2-kb promoter sequence of GsMYB15 was amplified by PCR (F, 5'-GGAGATAAAGCAAAC TTCTTGTTAC-3'; R, 5'-CTCATATCTGATGCTGTGTTGGC-3') using the genomic DNA of ED059 as the amplification template. The molecular evolutionary tree and phylogenetic analyses were performed using MEGA, version 5.0 [58]. The stress-related cis-acting elements of GsMYB15 were analyzed using the Plant-CARE database [59]. The protein sequence alignments were first assembled by Clustal W [60] and then, the aligned sequences were edited using BioEdit, version 7.0.4 (http: //www.mbio.ncsu.edu/bioedit/bioedit.html). The physicochemical properties of GsMYB15 were analyzed by the EXPASY online tools (https://web.expasy.org/protparam/).

\subsection{RNA Isolation and QPCR Analysis}

Total RNA was extracted from $0.2 \mathrm{~g}$ of fresh soybean leaves (ED059 and Tianlong 2), cotton bollworm, control Arabidopsis plants and T3 transgenic Arabidopsis plants that heterologously expressed GsMYB15. cDNA was synthesized using the First-Strain cDNA Synthesis SuperMix (Transgen Biotech, Beijing, China) according to the manufacturer's instructions. QPCR was performed as described by [3]. All the primers used for qPCR are listed in Supplementary Table S1. The relative expression level of each gene was quantified using the $2^{-\triangle \Delta \mathrm{Ct}}$ method [61].

\subsection{Sub-Cellular Localization and Transcriptional Activation Activity Analysis of GsMYB15}

The full-length coding sequence (CDS) of GsMYB15 was amplified using the following primer pair: F: 5'-ATCTGATCAAGAGACAGGATCCATGAGAACTCCATCATCTTCCTCTC-3'; R: 5'-GCCCTTGCTCACCATGGATCCTTGCAATAAGCCCACGTGCAAATC-3' (the underlined sequence were the same as the flanking sequences of the insertion cloning site of PJL12m-GFP). The PCR product was sub-cloned into the PJL12m-GFP vector using the LR method (ClonExpress ${ }^{\circledR}$ Entry One Step Cloning Kit, Vazyme, Nanjing, China) to generate PJL12m-GsMYB15-GFP. The GsMYB15-GFP fusion construct was under the control of the CaMV35S promoter. The construct was then transformed into Agrobacterium strain EHA105 using the method reported by [62].

Nicotiana tabacum 'benthamiana' plants were grown in a plant growth chamber at $26^{\circ} \mathrm{C}$ with a $16 \mathrm{~h} \mathrm{light} / 8 \mathrm{~h}$ dark regimen until plants that were approximately $10-20 \mathrm{~cm}$ high were available for infiltration with Agrobacterium strain EHA105. Infiltration was performed as described by [63]. 
The agroinfiltrated leaves were photographed 2 days after infiltration. GFP fluorescence images were captured with a Nikon ECLIPSE Ti laser scanning confocal microscope with excitation at $488 \mathrm{~nm}$.

To assess the transcriptional activation activity of GsMYB15, the full-length CDS of GsMYB15 was cloned into the bait vector pGBKT7 (BD) using the following primer pair: $F$ : 5'-AGGAC CTGCATATGGCCATGGAGATGAGAACTCCATCTTCCTCTCACAA-3'; R: 5' -CCGGGA ATTCGGCCTCCATGGTCATTGCAATAAGCCCACGTG- $3^{\prime}$ (the underlined sequence were the same as the flanking sequences of the insertion cloning site of pGBKT7). The detailed method for the generation of pGBKT7-GsMYB15 was the same as that for PJL12m-GsMYB15-GFP. The vector pGBKT7 (BD) was used as a negative control and the vector pGAL4 was used as a positive control. Yeast two-hybrid (Y2H) assessment was performed as described by [16].

\subsection{GUS Staining and Expression Analysis}

The 2-kb promoter sequence upstream of the start codon (ATG) of GsMYB15 was amplified from ED059 genomic DNA by PCR using the following primer pair: F: 5'-GACCTGCAGGCA TGCAAGCTTCGTTGGGGGTAGATATCGAATC-3'; R: 5'-TTACCCTCAGATCTACCATGGATCTGA TGCTGTGTTGGCGATG-3' (the underlined sequence were the same as the flanking sequences of insertion cloning site of pCambia3301-GUS). The PCR product was then introduced into the pCambia3301 binary vector using the LR method to replace the CaMV35S promoter and create GUS fusion constructs. The pro GsMYB15::GUS construct was then transformed into wild-type Arabidopsis and transgenic plants were selected by resistance to Basta. The $\mathrm{T}_{3}$ single-copy insertion transgenic lines were selected by the method described by [62]. The T3 transgenic plants were used for GUS staining as reported by [48].

\subsection{Generation of GsMYB15 Transgenic Arabidopsis Plants}

The full-length CDS of GsMYB15 was sub-cloned into the binary vector PB2GW7.0 and used to transform Agrobacterium strain EHA105. The transgenic Arabidopsis plants were transformed with EHA105 by the floral dip method [64]. $\mathrm{T}_{1}$ transgenic plants were grown in soil at $21^{\circ} \mathrm{C}$ in a plant growth chamber with a $16 \mathrm{~h}$ day $/ 8 \mathrm{~h}$ night regimen and selected by resistance to Basta. In addition, we also use RT-PCR and immunostrip (Bar Fast Immunostrip Kit, OCRI, Whuhan, China) methods to confirm the generations of GsMYB15 transgenic Arabidopsis. The $\mathrm{T}_{3}$ single-copy insertion transgenic lines were selected by the method described by [61].

\subsection{Tissue Preparation}

Paraffin sectioning was performed as described by [65].

\subsection{Analysis of Salinity Tolerance and Insect Resistance in Transgenic Arabidopsis Plants}

For germination analysis, at least 400 seeds per plate from the Control (Col-0), GsMYB15-OE2 (L-2), GsMYB15-OE3 (L-3) and GsMYB15-OE4 (L-4) Arabidopsis plants were sown onto MS medium supplemented with 0 or $100 \mathrm{mM} \mathrm{NaCl}$. Germination was assessed from 3 to 7 days after exposure to light. A seed was considered to be germinated when the radical protruded through the envelope. All treatments were performed in biological triplicates.

For salinity stress treatment, five-week-old, nutritional soil-grown, control Arabidopsis plants and Arabidopsis plants overexpressing GsMYB15 were irrigated with 0, 150, or $250 \mathrm{mM} \mathrm{NaCl}$ solution for 7 days and then with distilled water for 4 days. At the end of the treatment, the leaves were collected and immediately frozen in liquid nitrogen and stored at $-80{ }^{\circ} \mathrm{C}$ until further analysis. All the treatments were performed in biological triplicates.

For insect resistance analysis, $H$. armigera larvae were hatched at $28^{\circ} \mathrm{C}$ from a single egg mass obtained from Huazhong Agricultural University. For whole-plant feeding, each pot of the Arabidopsis plants contained fifteen freshly hatched individual $H$. armigera larvae and covered with a mesh bag to contain the larvae. For leaf feeding, detached leaves from control or transgenic plants were placed 
in a plastic bowl; each plastic bowl contained fifteen freshly hatched individual H. armigera larvae. After feeding for 7 days, increases in net weight were recorded. All treatments were performed in biological triplicates.

\section{Conclusions}

In conclusion, we demonstrated that GsMYB15 is localized to the nucleus and had transcriptional activation activity. Moreover, we describe that the expression of GsMYB15 could be induced by $\mathrm{NaCl}, \mathrm{MeJA}$, SA and Helicoverpa Armigera treatments. We also suggested that GsMYB15 from wild soybean (Glycine soja) could increase Helicoverpa Armigera resistance and salt tolerance in transgenic Arabidopsis plants.

Supplementary Materials: Supplementary Materials can be found at http:/ / www.mdpi.com/1422-0067/19/12/ 3958/s1.

Author Contributions: Conceptualization, X.-J.S. and Y.-Y.W.; Methodology, W.G.; Software, W.G.; Validation, Y.-X.Z., Y.-Y.W. and X.-J.S.; Formal analysis, Y.-Y.W.; Investigation, X.-J.S.; Resources, X.-A.Z.; Data curation, X.-J.S.; Writing-original draft preparation, X.-A.Z.; Writing—review and editing, X.-J.S.; Supervision, W.G.; Project administration, Y.-Y.W. and Y.-Q.J.

Funding: This research was funded by National Natural Science Foundation of China (No. 31501655) awarded to X.S.

Acknowledgments: We would like to give heartfelt thanks to Lu Qin for her kindly help and technical advice.

Conflicts of Interest: The authors declare no conflict of interest.

\section{Abbreviations}

$\begin{array}{ll}\text { MeJA } & \text { Methyl jasmonate } \\ \text { ABA } & \text { Abscisic acid } \\ \text { GUS } & \text { Beta-glucuronidase } \\ \text { TF } & \text { Transcription factor } \\ \text { SA } & \text { Salicylic Acid } \\ \text { bHLH } & \text { Basic helix-loop-helix } \\ \text { H. armigera } & \text { Helicoverpa armigera } \\ \text { OE } & \text { Overexpression }\end{array}$

\section{References}

1. Zhu, J.K. Salt and drought stress signal transduction in plants. Annu. Rev. Plant Biol. 2002, 53, $247-273$. [CrossRef] [PubMed]

2. Howe, G.; Jander, G. Plant immunity to insect herbivores. Annu. Rev. Plant Biol. 2008, 59, 41-66. [CrossRef] [PubMed]

3. Shen, X.J.; Guo, X.W.; Guo, X.; Zhao, D.; Zhao, W.; Chen, J.S.; Li, T.H. PacMYBA, a sweet cherry R2R3-MYB transcription factor, is a positive regulator of salt stress tolerance and pathogen resistance. Plant Physiol. Biochem. 2017, 112, 302-311. [CrossRef] [PubMed]

4. Chen, W.J.; Zhu, T. Networks of transcription factors with roles in environmental stress response. Trends Plant Sci. 2004, 9, 591-596. [CrossRef] [PubMed]

5. Hirayama, T.; Shinozaki, K. Research on plant abiotic stress responses in the post-genome era: Past, present and future. Plant J. 2010, 61, 1041-1052. [CrossRef] [PubMed]

6. Zhu, J.K. Abiotic stress signaling and responses in plants. Cell 2016, 167, 313-324. [CrossRef] [PubMed]

7. Melcher, K.; Ng, L.M.; Zhou, X.E.; Soon, F.F.; Xu, Y.; Suino-Powell, K.M.; Park, S.Y.; Weiner, J.J.; Fujii, H.; Chinnusamy, V.; et al. A gate-latch-lock mechanism for hormone signalling by abscisic acid receptors. Nature 2009, 462, 602-608. [CrossRef]

8. Walter, P.; Ron, D. The unfolded protein response: From stress pathway to homeostatic regulation. Science 2011, 334, 1081-1086. [CrossRef]

9. Endler, A.; Kesten, C.; Schneider, R.; Zhang, Y.; Ivakov, A.; Froehlich, A.; Funke, N.; Persson, S. A mechanism for sustained cellulose synthesis during salt stress. Cell 2015, 162, 1353-1364. [CrossRef] 
10. Nakashima, K.; Ito, Y.; Yamaguchi-Shinozaki, K. Transcriptional regulatory networks in response to abiotic stresses in Arabidopsis and grasses. Plant Physiol. 2009, 149, 88-95. [CrossRef]

11. Swarbreck, S.M.; Colaço, R.; Davies, J.M. Plant calcium-permeable channels. Plant Physiol. 2013, 163, 514-522. [CrossRef] [PubMed]

12. Leng, P.; Yuan, B.; Guo, Y. The role of abscisic acid in fruit ripening and responses to abiotic stress. J. Exp. Bot. 2014, 65, 4577-4588. [CrossRef] [PubMed]

13. Zheng, X.Z.; Kang, S.; Jing, Y.P.; Ren, Z.J.; Li, L.G.; Zhou, J.M.; Berkowitz, G.; Shi, J.S.; Fu, A.G.; Lan, W.Z.; et al. Danger-associated peptides close stomata by OST1-independent 3 activation of anion channels in guard cells. Plant Cell 2018, 30, 1132-1146. [CrossRef] [PubMed]

14. Yang, D.H.; Hettenhausen, C.; Baldwin, I.T.; Wu, J.J. Silencing nicotiana attenuata calcium-dependent protein kinases, CDPK4 and CDPK5, strongly up-regulates wound- and herbivory-induced jasmonic acid accumulations. Plant Physiol. 2012, 159, 1591-1607. [CrossRef] [PubMed]

15. Mao, Y.B.; Liu, Y.Q.; Chen, D.Y.; Chen, F.Y.; Fang, X.; Hong, G.J.; Wang, W.J.; Wang, W.J.; Chen, X.Y. Jasmonate response decay and defense metabolite accumulation contributes to age-regulated dynamics of plant insect resistance. Nat. Commun. 2015, 8, 13925. [CrossRef] [PubMed]

16. Shen, X.J.; Guo, X.; Zhao, D.; Zhang, Q.; Jiang, Y.Z.; Wang, Y.T.; Peng, X.; Wei, Y.; Zhai, Z.F.; Zhao, W.; et al. Cloning and expression profiling of the PacSnRK2 and PacPP2C gene families during fruit development, ABA treatment, and dehydration stress in sweet cherry. Plant Physiol. Biochem. 2017, 119, 275-285. [CrossRef] [PubMed]

17. Chico, J.M.; Fernández-Barbero, G.; Chini, A.; Fernández-Calvo, P.; Díez-Díaz, M.; Solano, R. Repression of Jasmonate-dependent defenses by shade involves differential regulation of protein stability of MYC transcription factors and their JAZ repressors in Arabidopsis. Plant Cell 2014, 26, 1967-1980. [CrossRef]

18. Louis, J.; Basu, S.; Varsani, S.; Castano-Duque, L.; Jiang, V.; Williams, W.P.; Felton, G.W.; Luthe, D.S. Ethylene Contributes to maize insect resistance1-Mediated Maize Defense against the Phloem Sap-Sucking Corn Leaf Aphid. Plant Physiol. 2015, 169, 313-324. [CrossRef]

19. Voght, S.P.; Fluegel, M.L.; Andrews, L.A.; Pallanck, L.J. Drosophila NPC1b Promotes an Early Step in Sterol Absorption from the Midgut Epithelium. Cell Metab. 2007, 5, 195-205. [CrossRef]

20. Verma, V.; Ravindran, P.; Kumar, P.P. Plant hormone-mediated regulation of stress responses. BMC Plant Biol. 2016, 16, 86. [CrossRef]

21. Hou, X.; Lee, L.Y.; Xia, K.; Yan, Y.; Yu, H. DELLAs modulate jasmonate signaling via competitive binding to JAZs. Dev. Cell 2010, 19, 884-894. [CrossRef] [PubMed]

22. Choi, J.; Huh, S.U.; Kojima, M.; Sakakibara, H.; Paek, K.H.; Hwang, I. The cytokinin-activated transcription factor ARR2 promotes plant immunity via TGA3/NPR1-dependent salicylic acid signaling in Arabidopsis. Dev. Cell 2010, 19, 284-295. [CrossRef] [PubMed]

23. Seo, P.J.; Park, C.M. MYB96-mediated abscisic acid signals induce pathogen resistance response by promoting salicylic acid biosynthesis in Arabidopsis. New Phytol. 2010, 186, 471-483. [CrossRef]

24. Li, R.; Zhang, J.; Li, J.C.; Zhou, G.X.; Wang, Q.; Bian, W.B.; Matthias, E.; Lou, Y.G. Prioritizing plant defence over growth through WRKY regulation facilitates infestation by non-target herbivores. eLife 2015, 4, E04805. [CrossRef] [PubMed]

25. Zhou, J.; Wang, J.; Zheng, Z.Y.; Fan, B.F.; Yu, J.Q.; Chen, Z.X. Characterization of the promoter and extended C-terminal domain of Arabidopsis WRKY33 and functional analysis of tomato WRKY33 homologues in plant stress responses. J. Exp. Bot. 2015, 66, 4567-4583. [CrossRef]

26. Guo, J.P.; Xu, C.X.; Wu, D.; Zhao, Y.; Qiu, Y.F.; Wang, X.X.; OuYang, Y.D.; Cai, B.D.; Liu, X.; Jing, S.L.; et al. Bph6 encodes an exocyst-localized protein and confers broad resistance to planthoppers in rice. Nat. Genet. 2018, 50, 297-306. [CrossRef] [PubMed]

27. Zhong, Y.J.; Wang, Y.G.; Guo, J.F.; Zhu, X.L.; Shi, J.; He, Q.J.; Liu, Y.; Wu, Y.R.; Zhang, L.; Lv, Q.D.; et al. Rice SPX6 negatively regulates the phosphate starvation response through suppression of the transcription factor PHR2. New Phytol. 2018, 219, 135-148. [CrossRef] [PubMed]

28. Dubos, C.; Stracke, R.; Grotewold, E.; Weisshaar, B.; Martin, C.; Lepiniec, L. MYB transcription factors in Arabidopsis. Trends Plant Sci. 2010, 15, 573-581. [CrossRef]

29. Wang, T.; Tohge, T.; Ivakov, A.; Mueller-Roeber, B.; Fernie, A.R.; Mutwil, M.; Schippers, J.H.; Persson, S. Salt-related MYB1 coordinates abscisic acid biosynthesis and signaling during salt stress in Arabidopsis. Plant Physiol. 2015, 169, 1027-1041. [CrossRef] 
30. Wei, Q.H.; Zhang, F.; Sun, F.S.; Luo, Q.C.; Wang, R.B.; Chen, M.J.; Chen, M.J.; Chang, J.L.; Yang, G.X.; He, G.Y. A wheat MYB transcriptional repressor TaMyb1D regulates phenylpropanoid metabolism and enhances tolerance to drought and oxidative stresses in transgenic tobacco plants. Plant Sci. 2017, 265, 112-123. [CrossRef]

31. Seo, P.J.; Lee, S.B.; Suh, M.C.; Park, M.J.; Go, Y.S.; Park, C.M. The MYB96 Transcription factor regulates cuticular wax biosynthesis under drought conditions in Arabidopsis. Plant Cell 2011, 23, 1138-1152. [CrossRef] [PubMed]

32. Zhai, Y.; Li, P.; Mei, Y.; Chen, M.Y.; Chen, X.C.; Xu, H.; Zhou, X.A.; Dong, H.S.; Zhang, C.L.; Jiang, W.H. Three MYB genes co-regulate the phloem-based defence against English grain aphid in wheat. J. Exp. Bot. 2017, 15, 4153-4169. [CrossRef] [PubMed]

33. Onkokesung, N.; Reichelt, M.; van Doorn, A.; Schuurink, R.C.; van Loon, J.J.; Dicke, M. Modulation of flavonoid metabolites in Arabidopsis thaliana through overexpression of the MYB75 transcription factor: Role of kaempferol-3,7dirhamnoside in resistance to the specialist insect herbivore Pieris brassicae. J. Exp. Bot. 2014, 65, 2203-2217. [CrossRef] [PubMed]

34. Mourtzinis, S.; Borg, B.S.; Naeve, S.L.; Osthus, J.; Conley, S.P. Characterizing Soybean Meal Value Variation across the United States: A Swine Case Study. Agron. J. 2018, 110, 2343-2349. [CrossRef]

35. Olmstead, J.; Brummer, E.C. Benefits and barriers to perennial forage crops in Iowa corn and soybean rotations. Renew. Agric. Food Syst. 2008, 23, 97-107. [CrossRef]

36. Ullah, A.; Manghwar, H.; Shaban, M.; Khan, A.H.; Akbar, A.; Ali, U.; Ali, E.; Fahad, S. Phytohormones enhanced drought tolerance in plants: A coping strategy. Environ. Sci. Pollut. Res. 2018, 25, 33103-33118. [CrossRef] [PubMed]

37. Parmar, N.; Singh, K.H.; Sharma, D.; Singh, L.; Kumar, P.; Nanjundan, J.; Khan, Y.J.; Chauhan, D.K.; Thakur, A.K. Genetic engineering strategies for biotic and abiotic stress tolerance and quality enhancement in horticultural crops: A comprehensive review. 3 Biotech 2017, 7, 239. [CrossRef]

38. Espley, R.V.; Hellens, R.P.; Putterill, J.; Stevenson, D.E.; Kutty-Amma, S.; Allan, A.C. Red colouration in apple fruit is due to the activity of the MYB transcription factor, MdMYB10. Plant J. 2007, 49, 414-427. [CrossRef]

39. Jakab, G.; Ton, J.; Flors, V.; Zimmerli, L.; Metraux, J.P.; Mauch-Mani, B. Enhancing Arabidopsis salt and drought stress tolerance by chemical priming for its abscisic acid responses. Plant Physiol. 2005, 139, $267-274$. [CrossRef]

40. Chen, J.H.; Jiang, H.W.; Hsieh, E.J.; Chen, H.Y.; Chien, C.T.; Hsieh, H.L.; Lin, T.P. Drought and salt stress tolerance of Arabidopsis glutathione S-transferase U17 knockout mutant are attributed to the combined effect of glutathione and abscisic acid. Plant Physiol. 2011, 158, 340-351. [CrossRef]

41. Browse, J. Jasmonate Passes Muster: A receptor and targets for the defense hormone. Annu. Rev. Plant Biol. 2009, 60, 183-205. [CrossRef] [PubMed]

42. Yan, C.; Fan, M.; Yang, M.; Zhao, J.J.; Zhang, W.B.; Su, Y.; Xiao, L.L.; Deng, H.T.; Xie, D.X. Injury Activates $\mathrm{Ca}^{2+} /$ Calmodulin-Dependent Phosphorylation of JAV1-JAZ8-WRKY51 Complex for Jasmonate Biosynthesis. Mol. Cell 2018, 70, 136-149. [CrossRef] [PubMed]

43. Spoel, S.H.; Johnson, J.S.; Dong, X. Regulation of tradeoffs between plant defenses against pathogens with different life styles. Proc. Natl. Acad. Sci. USA 2007, 104, 18842-18847. [CrossRef]

44. Abe, H.A.; Urao, T.U.; Ito, T.; Seki, M.; Shinozaki, K.; Yamaguchi-Shinozaki, K. Arabidopsis AtMYC2 (bHLH) and AtMYB2 (MYB) Function as transcriptional activators in abscisic acid signaling. Plant Cell 2003, 15, 63-78. [CrossRef] [PubMed]

45. Jiang, Y.Q.; Deyholos, M.K. Functional characterization of Arabidopsis NaCl-inducible WRKY25 and WRKY33 transcription factors in abiotic stresses. Plant Mol. Biol. 2009, 69, 91-105. [CrossRef]

46. Chung, Y.; Kwon, S.I.; Choe, S.H. Antagonistic regulation of Arabidopsis growth by brassinosteroids and abiotic stresses. Mol. Cells 2014, 37, 795-803. [CrossRef]

47. Pruthvi, V.; Narasimhan, R.; Nataraja, K.N. Simultaneous expression of abiotic stress responsive transcription factors, AtDREB2A, AtHB7 and AtABF3 improves salinity and drought tolerance in peanut (Arachis hypogaea L.). PLoS ONE 2014, 9, E111152. [CrossRef]

48. Ding, Z.J.; Yan, J.Y.; Li, C.X.; Li, G.X.; Wu, Y.R.; Zheng, S.J. Transcription factor WRKY46 modulates the development of Arabidopsis lateral roots in osmotic/salt stress conditions via regulation of ABA signaling and auxin homeostasis. Plant J. 2015, 84, 56-69. [CrossRef] 
49. Wu, J.Q.; Baldwin, I.T. New insights into plant responses to the attack from insect herbivores. Annu. Rev. Genet. 2010, 44, 1-24. [CrossRef]

50. An, J.P.; Li, R.; Qu, F.J.; You, C.X.; Wang, X.F.; Hao, Y.J. R2R3-MYB transcription factor MdMYB23 is involved in the cold tolerance and proanthocyanidin accumulation in apple. Plant J. 2018, 96, 562-577. [CrossRef]

51. Xu, F.C.; Liu, H.L.; Xu, Y.Y.; Zhao, J.R.; Guo, Y.W.; Long, L.; Gao, W.; Song, C. Heterogeneous expression of the cotton R2R3-MYB transcription factor GbMYB60 increases salt sensitivity in transgenic Arabidopsis. Plant Cell Tissue Organ Cult. 2017, 133, 15-25. [CrossRef]

52. Xie, Y.P.; Chen, P.X.; Yan, Y.; Bao, C.N.; Li, X.W.; Wang, L.P.; Shen, X.X.; Li, H.Y.; Liu, X.F.; Niu, C.D.; et al. An atypical R2R3 MYB transcription factor increases cold hardiness by CBF-dependent and CBF-independent pathways in apple. New Phytol. 2017, 218, 201-218. [CrossRef] [PubMed]

53. Guo, J.L.; Ling, H.; Ma, J.J.; Chen, Y.; Su, Y.C.; Lin, Q.L.; Gao, S.W.; Wang, H.B.; Que, Y.X.; Xu, L.P. A sugarcane R2R3-MYB transcription factor gene is alternatively spliced during drought stress. Sci. Rep. 2017, 7, 2045-2322. [CrossRef] [PubMed]

54. Li, T.; Zhang, X.Y.; Huang, Y.; Xu, Z.S.; Wang, F.; Xiong, A.S. An R2R3-MYB transcription factor, S1MYB28, involved in the regulation of TYLCV infection in tomato. Sci. Hortic. 2018, 237, 192-200. [CrossRef]

55. Spoel, S.H.; Koornneef, A.; Claessens, S.M.C.; Korzelius, J.P.; Van Pelt, J.A.; Mueller, M.J.; Bechala, A.J.; Metraux, J.P.; Brown, R.; Kazan, K.; et al. NPR1 Modulates Cross-Talk between Salicylate- and Jasmonate-Dependent defense pathways through a novel function in the cytosol. Plant Cell 2003, 15, 760-770. [CrossRef]

56. Wasternack, C. Jasmonates: An update on biosynthesis, signal transduction and action in plant stress response, growth and development. Ann. Bot. 2007, 100, 681-697. [CrossRef]

57. Koo, A.J.; Gao, X.; Jones, A.D.; Howe, G.A. A rapid wound signal activates the systemic synthesis of bioactive jasmonates in Arabidopsis. Plant J. 2009, 59, 974-986. [CrossRef]

58. Tamura, K.; Peterson, D.; Peterson, N.; Stecher, G.; Nei, M.; Kumar, S. MEGA5: Molecular evolutionary genetics analysis using maximum likelihood, evolutionary distance, and maximum parsimony methods. Mol. Biol. Evol. 2011, 28, 2731-2739. [CrossRef]

59. Lescot, M.; Déhais, P.; Thijs, G.; Marchal, K.; Moreau, Y.; Peer, Y.V. PlantCARE, a database of plant cis-acting regulator elements and a portal to tools for in silico analysis of promoter sequences. Nucleic Acids Res. 2002, 30, 325-327. [CrossRef]

60. Chenna, R. Multiple sequence alignment with the Clustal series of programs. Nucleic Acids Res. 2003, 31, 3497-3500. [CrossRef]

61. Livak, K.J.; Schmittgen, T.D. Analysis of relative gene expression data using real-time quantitative PCR and the $2^{-\Delta \Delta C t}$ Method. Methods 2001, 25, 402-408. [CrossRef]

62. Shen, X.; Zhao, K.; Liu, L.; Zhang, K.; Yuan, H.; Liao, X.; Wang, Q.; Guo, X.W.; Li, F.; Li, T.H. A role for PacMYBA in ABA-regulated anthocyanin biosynthesis in red-colored sweet cherry cv. Hong Deng (Prunus avium L.). Plant Cell Physiol. 2014, 55, 862-880. [CrossRef]

63. Bai, G.; Yang, D.H.; Zhao, Y.; Ha, S.; Yang, F.; Ma, J.; Gao, X.S.; Wang, Z.M.; Zhu, J.K. Interactions between soybean ABA receptors and type 2C protein phosphatases. Plant Mol. Biol. 2013, 83, 651-664. [CrossRef]

64. Steven, J.C.; Andrew, F.B. Floral dip: A simplified method for Agrobacterium-mediated transformation of Arabidopsis thaliana. Plant J. 1998, 16, 735-743. [CrossRef]

65. Kerk, N.M.; Ceserani, T.; Tausta, S.L.; Sussex, I.M.; Nelson, T.M. Laser capture microdissection of cells from plant tissues. Plant Physiol. 2003, 132, 27-35. [CrossRef]

(C) 2018 by the authors. Licensee MDPI, Basel, Switzerland. This article is an open access article distributed under the terms and conditions of the Creative Commons Attribution (CC BY) license (http://creativecommons.org/licenses/by/4.0/). 